\title{
Watermarking Application Using Bit Plane Allocation
}

\author{
Gwanggil Jeon \\ Department of Embedded Systems Engineering, Incheon National University \\ 119 Academy-ro, Yeonsu-gu, Incheon 406-772, Korea \\ gjeon@incheon.ac.kr
}

\begin{abstract}
This paper studies about bit plane watermarking. The watermarking has been studied to protect digital image against illegal copyright action. Generally speaking, adding watermarking symbol in least significant bit (LSB) is desirable. However, sometimes watermarking information in LSB can be lost during the compression and transmission. Therefore, well designed watermarking technique is necessary. In this paper, we show how to design watermarking application, and compare watermarking performance on different bit plane. Two datasets were used; they are Kodak and McM datasets.
\end{abstract}

Keywords: Watermark, bit plane slicing, color image

\section{Introduction}

Watermarking stands for ensuring data integrity which unites aspects of data hashing and digital watermarking [1-4]. The watermark is an identifiable pattern which seems as various shades of brightness when observed by transmitted light. Traditionally, the watermarks have been employed on postage stamps, currency, and some official documents to protect counterfeiting. As in other industry, watermarking is important in computer science field. The watermarking is a crucial protection approach to where message is concealed in the multimedia information [5-15]. In this work, we study about image watermarking on image processing.

The watermarking has been developed to defense digital image again against illegal copyright violation [16-24]. The best method is to guarantee to provide images with maximal fidelity and robust against different attacks [25-28]. In this paper we study watermarking with bit plane slicing. The bit plane of a digital image is a set of bits fit to a determined bit level in each of the binary numbers representing the signal. Therefore, there are many scenarios to generated watermarked images. There are some pros and cons of watermarking approach. Normally it is good to add watermarking symbol in the least significant bit (LSB), however the symbol may be removed or changed during the transmission because LSB can be ignored hardly or blurred during the compression. However, we cannot add watermarking symbol in the most significant bit (MSB) because it may degrade image quality. Therefore, this relation is trade-off.

This paper is arranged as followed. In Section 2, we present color image bit plane slicing process. Section 3 shows some metrics used for simulation comparison. Section 4 provides assessed performance results. Finally, Section 5 presents conclusions. 


\section{Color Image Bit Plane Slicing}

A bit plane slicing method is employed for separation of the raw information from black and white or color images into each bit plane. Figure 1 shows the process of proposed watermarking in bit plane slicing.

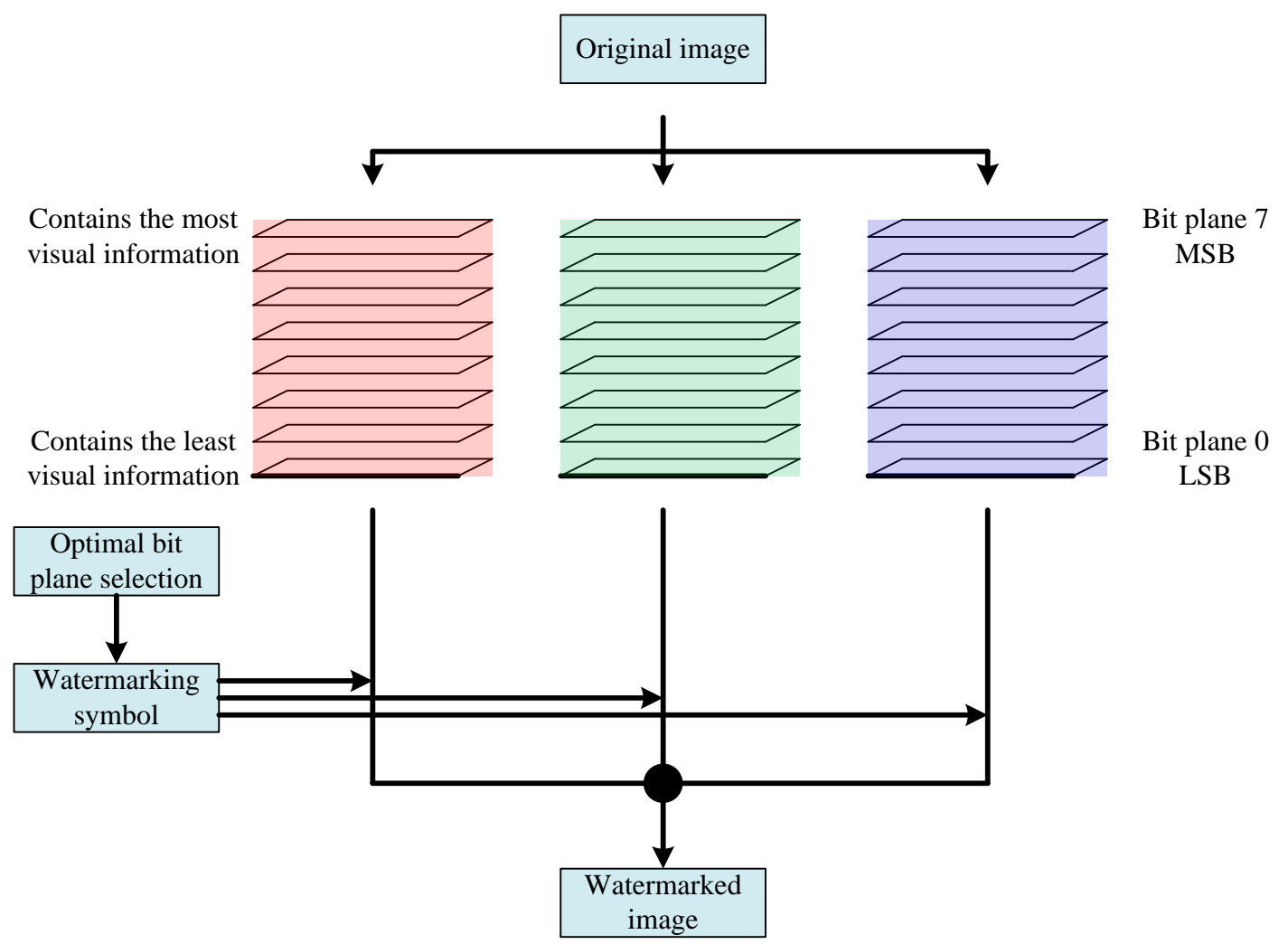

Figure 1. Bit Plane Slicing on Color Image

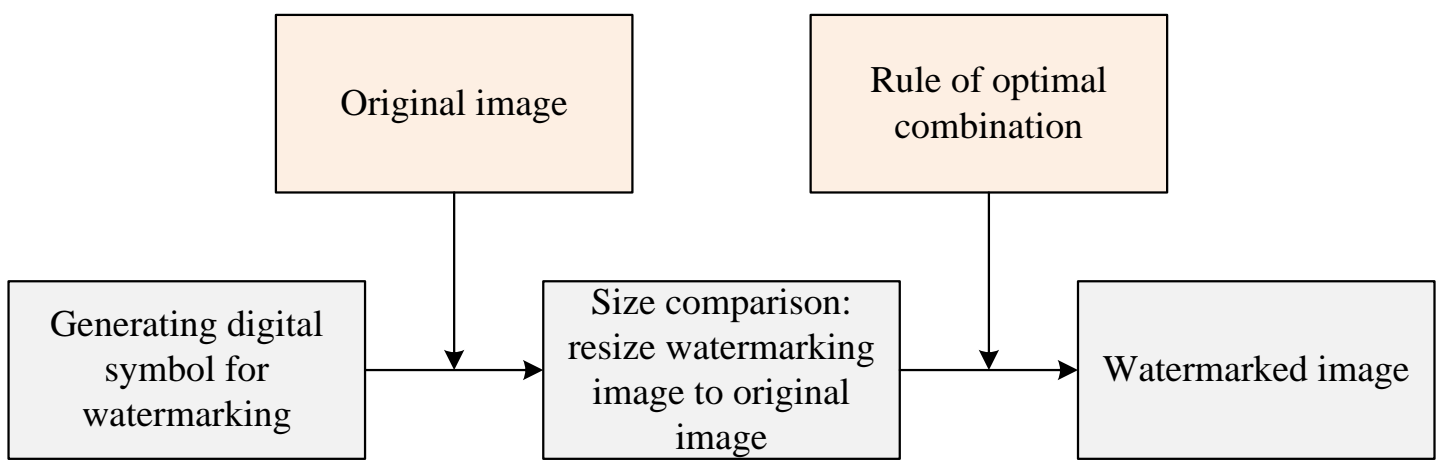

Figure 2. Watermarked Image Generation

We first select optimal bit plane out of eight candidates in each color channel, and add generated watermarking symbol in each channel. As information in LSB may be removed easily, it is requested to avoid using LSB information. The watermarking symbol is generated as shown in Figure 2. The generated watermarking symbol is added in the original image. As 
both images are not always with the same size, size comparison process is requested. After resizing watermarking symbol with the original image, symbol is merged with original image by the rule of optimal combination. Finally, watermarked images are obtained. Figure 3 shows the different bit plane images acquired using bit plane slice techniques on \#11 McM image.

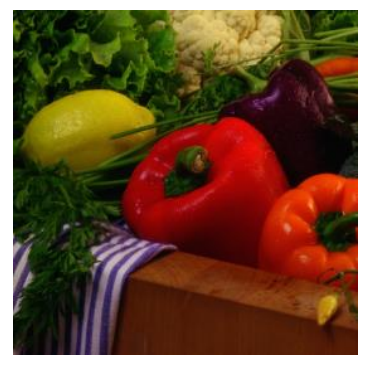

(a)

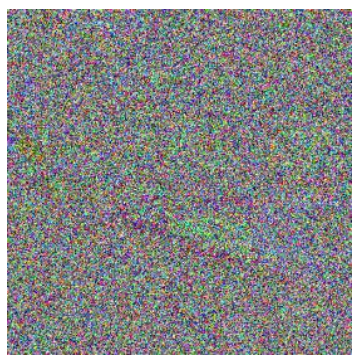

(b)

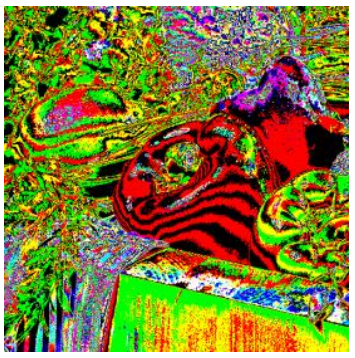

(f)

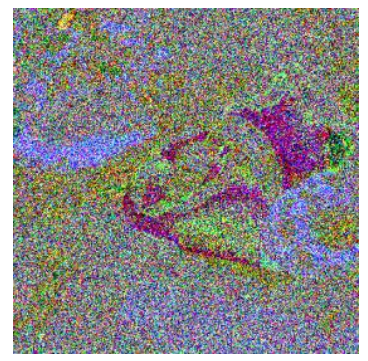

(c)

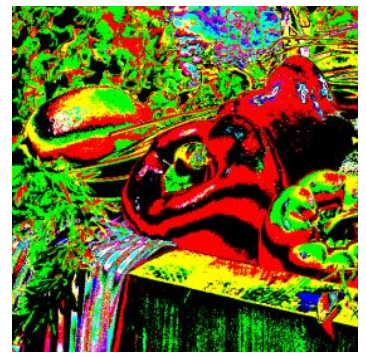

(g)

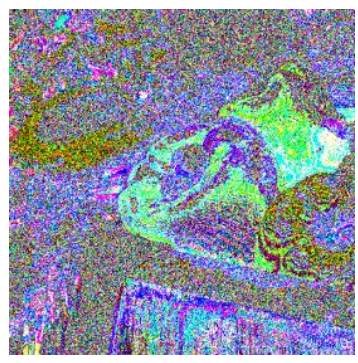

(d)

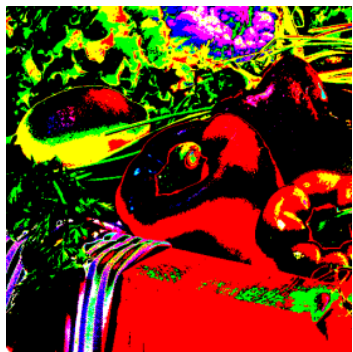

(h)

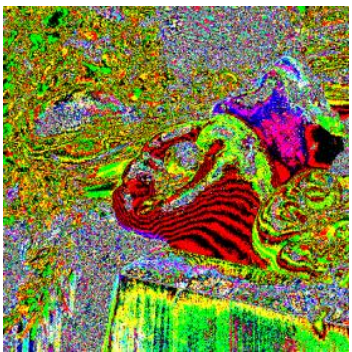

(e)

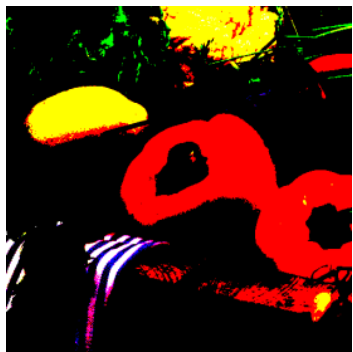

(i)

Figure 3. Bit Plane Slicing Example on \#11 McM Image: (a) Original Color Image, (b) $0^{\text {th }}$ Bit Plane LSB Image, (c) $1^{\text {st }}$ Bit Plane, (d) $2^{\text {nd }}$ Bit Plane, (e) $3^{\text {rd }}$ Bit Plane, (f) $4^{\text {th }}$ Bit Plane, (g) $5^{\text {th }}$ Bit Plane, (h) $6^{\text {th }}$ Bit Plane, and (i) $7^{\text {th }}$ Bit Plane MSB Image

It can be found from Figure 3 that, each image can be separated into eight images $\left(0^{\text {th }}\right.$ to $\left.7^{\text {th }}\right)$. Each bit takes different level of values, which affects the characteristics of each sliced image. From Figure 3, it is obvious that the most significant bit $\left(7^{\text {th }}\right)$ includes the clear manner to describe the image, and it can be utilized to descript the image. The other images $\left(0^{\text {th }}\right.$ to $\left.6^{\text {th }}\right)$ are not as clear as $7^{\text {th }}$ image, and sometimes those images seem to be noise. 


\section{Metrics for Evaluating Performance}

In this paper, we use three metrics for 10 objective perofmrance comaprison. First of all, peak signal-to-noise ratio (PSNR) is used which is for the ratio between the original maximum possible energy of a image and the energy of corrupting noise that reflect the fidelity of its state. In general, PSNR is used to evaluate the excellency of restored image. Normally the term, noise, stands for erorrs, the differnce between origianl and the reconstructed images. For $N$ bit images, the maximum value is $2^{N}-1$, therefore for $N=8$ bit image, the maximum value is $256-1=255$. The PSNR equeation is shown below:

$$
P S N R=20 \log _{10}(M A X)-10 \log _{10}(M S E),
$$

where MAX is 255.

We separated color image into three channels, therfore we can evaluate four different PSNR values: PSNR for red channel $\left(\mathrm{M}_{1}\right)$, PSNR for green channel $\left(\mathrm{M}_{2}\right)$, PSNR for blue channel $\left(\mathrm{M}_{3}\right)$, and color PSNR $\left(\mathrm{M}_{4}\right)$.

By the way, before obtaining PSNR, it is required to know MSE (mean squared error). The MSE is a metric to evaluate the average of the squares of the errors. Again, errors means the differnce between original and restored images. Given a noise-free $k \times l$ size monochrome image, MSE is obtained as,

$$
M S E=\frac{1}{k \times l} \sum_{x=0}^{k-1} \sum_{y=0}^{l-1}(\operatorname{ori}(x, y)-\operatorname{res}(x, y))^{2},
$$

where $\operatorname{ori}(x, y)$ and $\operatorname{res}(x, y)$ stand for original and restored pixels at the location of $(x, y)$.

We also use FSIM (Feature SIMilarity) for performance comparison. The FSIM index is defined as

$$
F S I M=\frac{\sum_{x \in \Omega} S_{L}(x) \cdot P C_{m}(x)}{\sum_{x \in \Omega} P C_{m}(x)},
$$

where $\Omega$ stands for the whole image, $\mathrm{PC}$ is phase congruency, $S_{L}(x)$ is,

$$
S_{L}(x)=\left[S_{P C}(x)\right]\left[S_{G}(x)\right] .
$$

The FSIM $_{\mathrm{C}}$ index is defined as

$$
F \operatorname{SIM}_{C}=\frac{\sum_{x \in \Omega} S_{P C}(x) S_{G}(x)\left[S_{I}(x) S_{Q}(x)\right]^{\lambda} \cdot P C_{m}(x)}{\sum_{x \in \Omega} P C_{m}(x)} .
$$

\section{Performance Evaluation}

The proposed method is tested in many different types of color iamges. The provided method was conducted using the MATLAB 2010. The used image for simulatio are shown in below Figures. Two testsets were employed: 24 Kodak imageset and $18 \mathrm{McM}$ imageset. They are shown in Figures 4 and 5. 


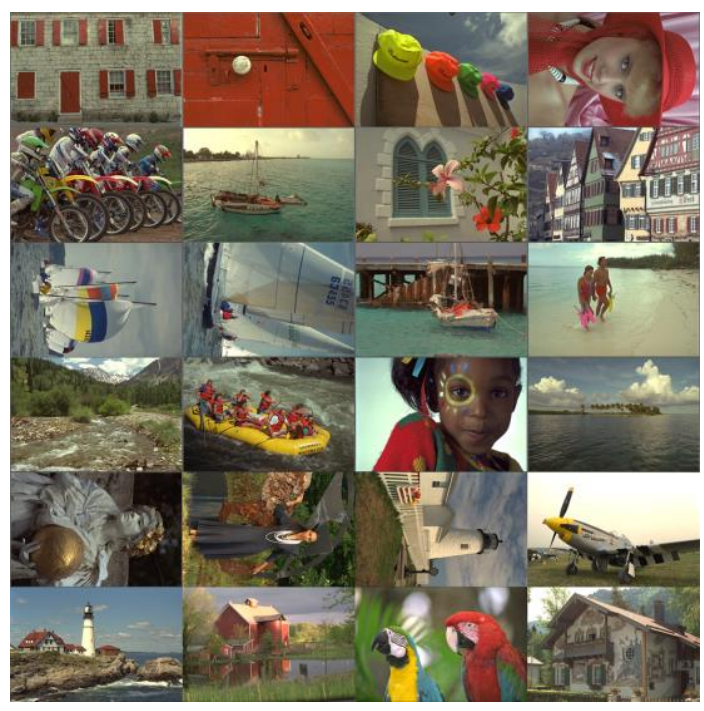

Figure 4. 24 Kodak Image Set

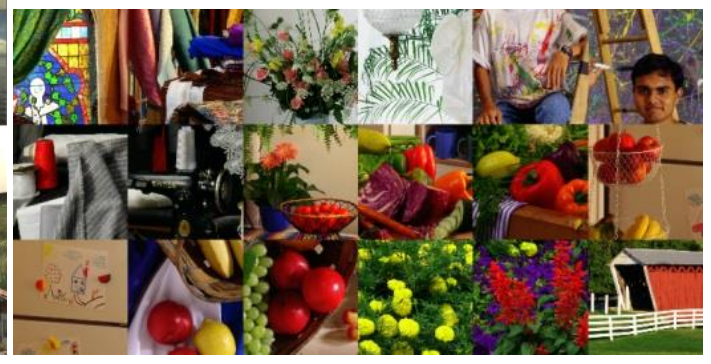

Figure 5. 18 McM Image Set

To conduct simulation we generated a symbol for watermarking. In this paper, the term 'Val is pretty!' is used for watermarking test, which is shown in Figure 6.

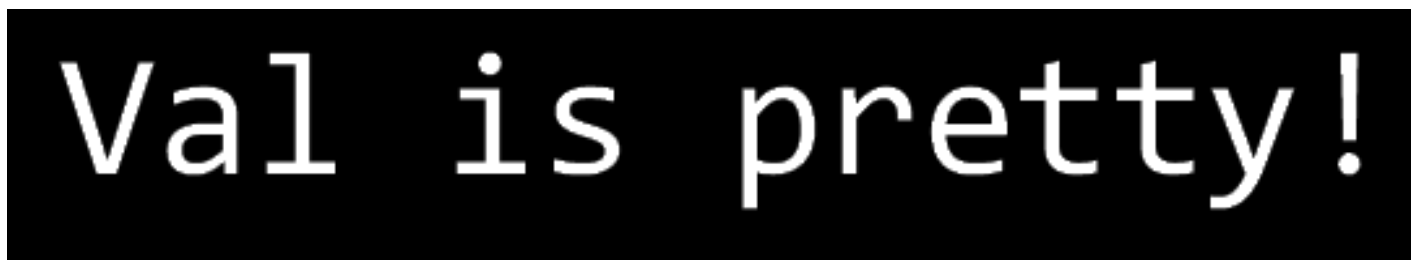

Figure 6. Test Text for Watermarking

The proposed method when applied to different Kodak and McM images provides the results which are shown Figure 7. All image in leftside are origianl images, and all image in rightside are watermarking added images. Figures 7(a), 7(b), 7(c), and 7(d) are watermarked images on \#5 Kodak, \#6 Kodak, \#10 McM, and \#11 McM, respectively.
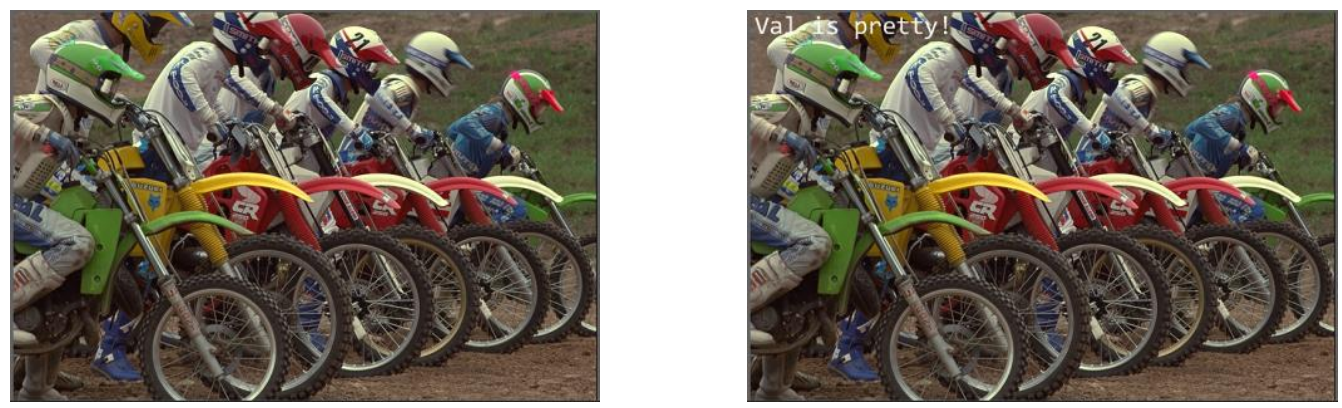

(a) 

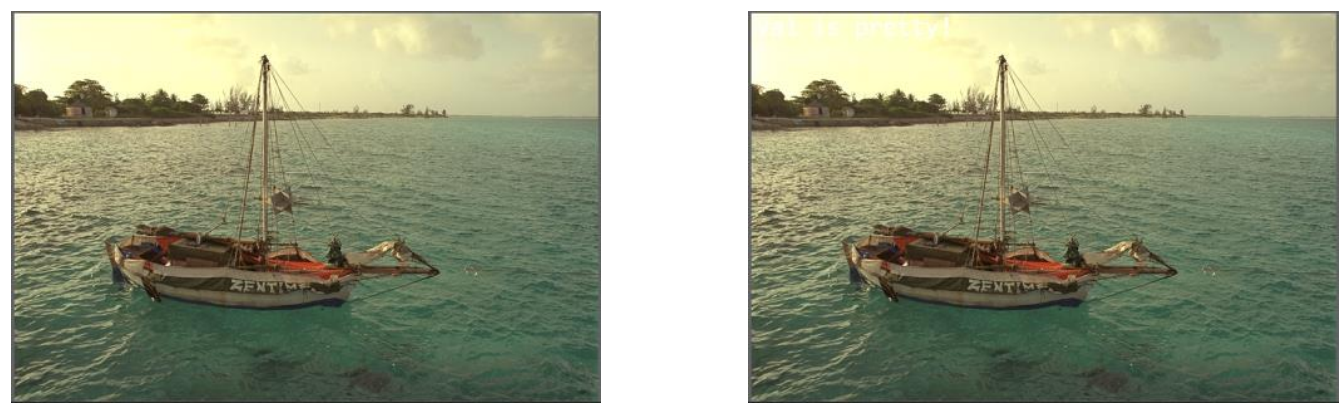

(b)
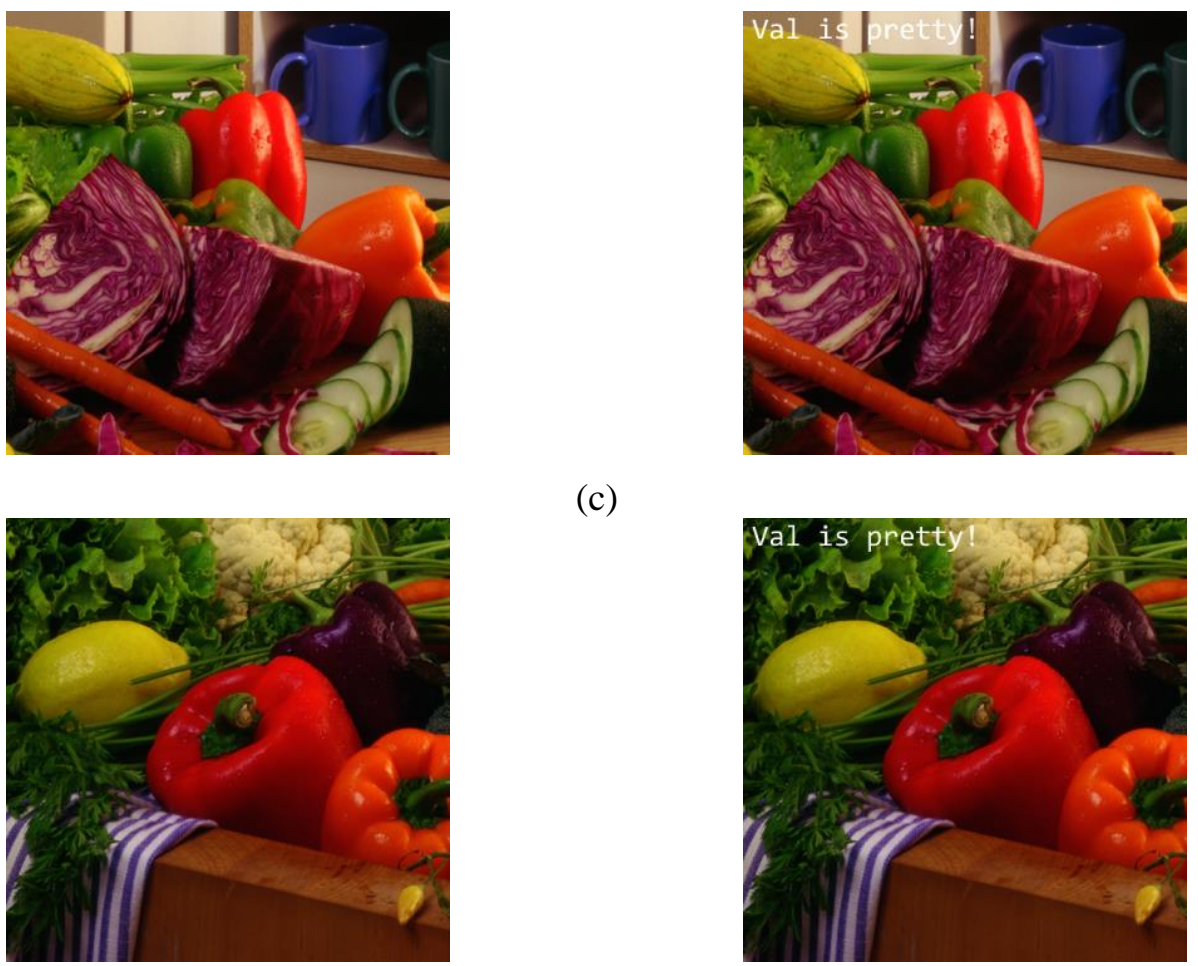

(c)

(d)

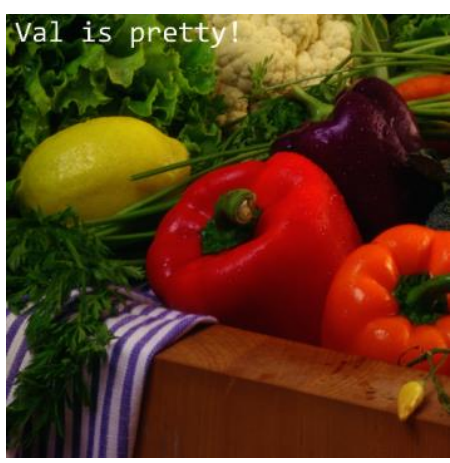

Figure 7. (Left Images) Original, (Right Images) Watermarked: (a) Kodak \#5, (b) Kodak \#6, (c) McM \#10, and (d) McM \#11 

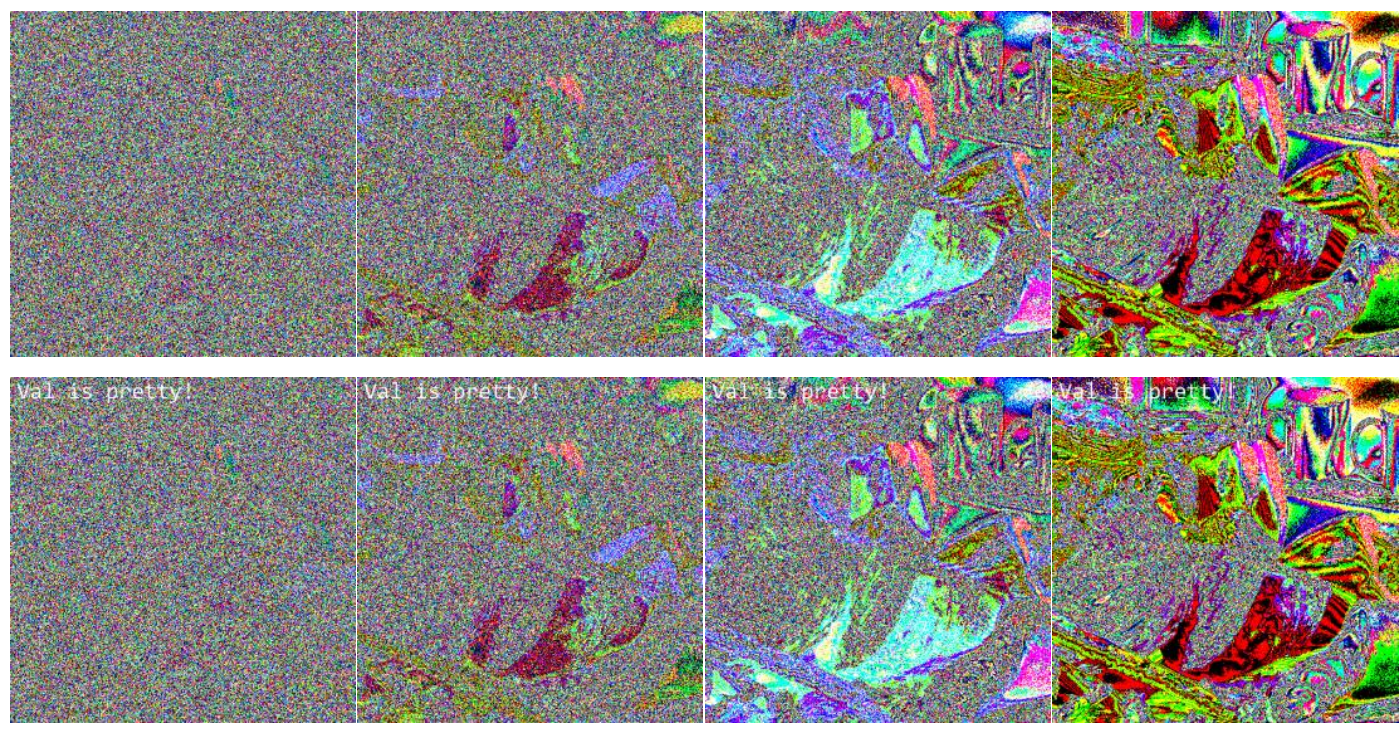

(a)

(b)

(c)

(d)
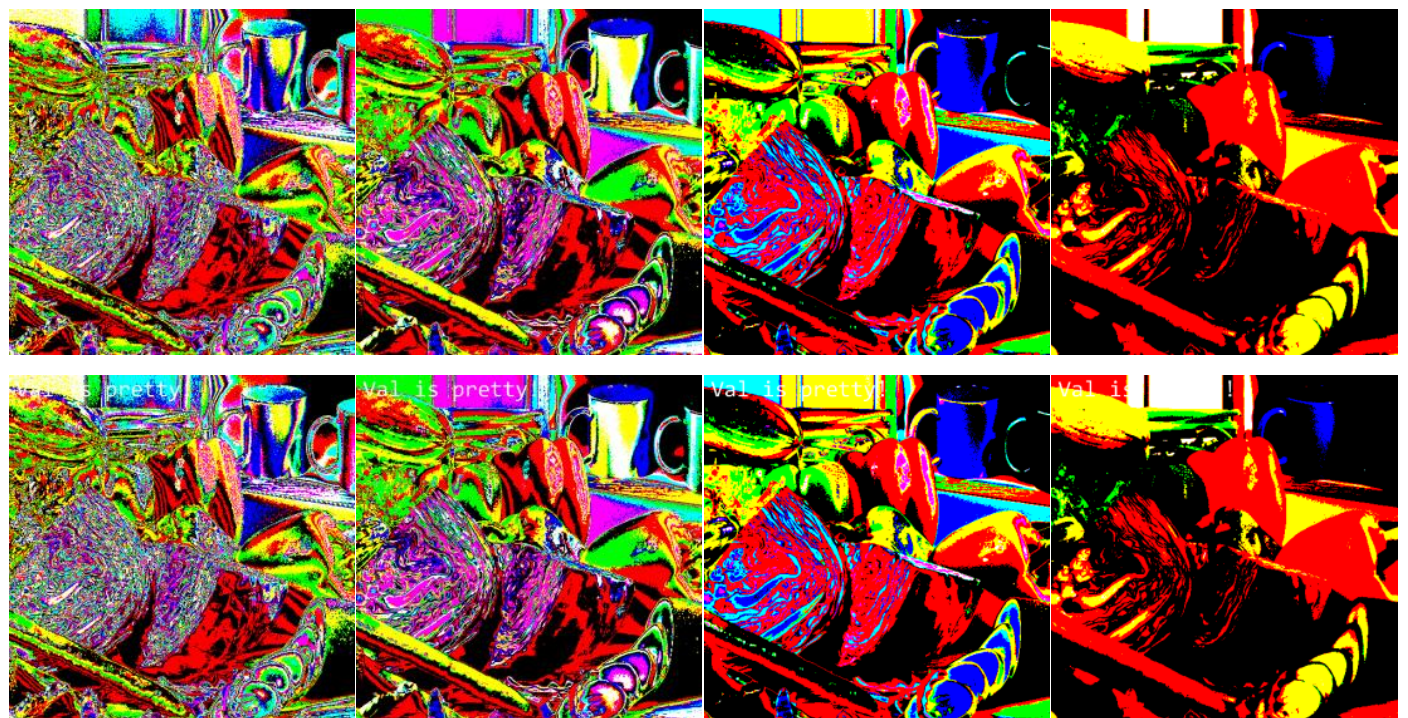

(e)

(f)

(g)

(h)

Figure 8. Watermarking Results on $0^{\text {th }}$ to $7^{\text {th }}$ Bit-planes

Figure 8 shows eight-level result images before and after the watermarking is applied. Each example consists of two images where upper-side images are without watermarking process and the images located lower-side are with watermarking process. As bit plane close to LSB looks like noise, it is more efficient to add watermarking symbol on lower planes.

As described in previous section, we use three metrics in this paper. They are MSE, PSNR and FSIM. Metrics $M_{1}$ to $M_{4}$ are from MSE, metrics $M_{5}$ to $M_{8}$ are from PSNR, and metrics $\mathrm{M}_{9}$ and $\mathrm{M}_{10}$ are FSIM results. Some descriptions are shown below:
$\mathrm{M}_{1}: \mathrm{MSE} \mathrm{R}$
$\mathrm{M}_{4}:$ CMSE_RGB
$\mathrm{M}_{7}:$ PSNR_B
$\mathrm{M}_{2}$ : MSE_G
$\mathrm{M}_{5}$ : PSNR_R
$M_{8}:$ CPSNR_RGB
$M_{3}$ : MSE_B
$M_{6}$ : PSNR_G
$\mathrm{M}_{9}:$ FSIM 


\section{$\mathrm{M}_{10}:$ FSIMC}

There are eight rows in each Table, which are arranged as,

$$
\begin{aligned}
& 1^{\text {st }} \text { row: watermarking on } 0^{\text {th }} \text { plane } \\
& 2^{\text {nd }} \text { row: watermarking on } 1^{\text {st }} \text { plane } \\
& 3^{\text {rd }} \text { row: watermarking on } 2^{\text {nd }} \text { plane } \\
& 4^{\text {th }} \text { row: watermarking on } 3^{\text {th }} \text { plane } \\
& 5^{\text {th }} \text { row: watermarking on } 4^{\text {th }} \text { plane } \\
& 6^{\text {th }} \text { row: watermarking on } 5^{\text {th }} \text { plane } \\
& 7^{\text {th }} \text { row: watermarking on } 6^{\text {th }} \text { plane } \\
& 8^{\text {th }} \text { row: watermarking on } 7^{\text {th }} \text { plane }
\end{aligned}
$$

Tables 1-4 show the performance comparison on \#5 Kodak, \#6 Kodak, \#10 McM, and \#11 McM images.

Table 1. Performance Comparison on \#5 Kodak Image

\begin{tabular}{|r|r|r|r|c|c|c|c|c|c|}
\hline \multicolumn{1}{|c|}{$\mathrm{M}_{1}$} & \multicolumn{1}{c|}{$\mathrm{M}_{2}$} & \multicolumn{1}{|c|}{$\mathrm{M}_{3}$} & \multicolumn{1}{c|}{$\mathrm{M}_{4}$} & $\mathrm{M}_{5}$ & $\mathrm{M}_{6}$ & $\mathrm{M}_{7}$ & $\mathrm{M}_{8}$ & $\mathrm{M}_{9}$ & $\mathrm{M}_{10}$ \\
\hline 91.9 & 93.6 & 98.8 & 94.8 & 28.50 & 28.42 & 28.18 & 28.36 & 0.9852 & 0.9842 \\
\hline 99.7 & 92.1 & 95.4 & 95.7 & 28.14 & 28.49 & 28.34 & 28.32 & 0.9842 & 0.9831 \\
\hline 94.1 & 91.4 & 100.1 & 95.2 & 28.39 & 28.52 & 28.13 & 28.34 & 0.9869 & 0.9858 \\
\hline 90.1 & 87.5 & 102.1 & 93.2 & 28.58 & 28.71 & 28.04 & 28.44 & 0.9911 & 0.9902 \\
\hline 102.8 & 66.6 & 100.9 & 90.1 & 28.01 & 29.90 & 28.09 & 28.58 & 0.9957 & 0.9953 \\
\hline 72.1 & 123.5 & 141.9 & 112.5 & 29.55 & 27.22 & 26.61 & 27.62 & 0.9958 & 0.9955 \\
\hline 66.0 & 55.5 & 90.5 & 70.7 & 29.94 & 30.69 & 28.56 & 29.64 & 0.9966 & 0.9965 \\
\hline 138.6 & 155.3 & 160.7 & 151.5 & 26.71 & 26.22 & 26.07 & 26.33 & 0.9881 & 0.9880 \\
\hline
\end{tabular}

Table 2. Performance Comparison on \#6 Kodak image

\begin{tabular}{|c|c|c|c|c|c|c|c|c|c|}
\hline $\mathrm{M}_{1}$ & $\mathrm{M}_{2}$ & $\mathrm{M}_{3}$ & $\mathrm{M}_{4}$ & $\mathrm{M}_{5}$ & $\mathrm{M}_{6}$ & $\mathrm{M}_{7}$ & $\mathrm{M}_{8}$ & $\mathrm{M}_{9}$ & $\mathrm{M}_{10}$ \\
\hline 0.8 & 3.4 & 96.5 & 33.6 & 48.97 & 42.76 & 28.29 & 32.87 & 0.9987 & 0.9985 \\
\hline 0.6 & 3.3 & 95.2 & 33.0 & 50.65 & 42.91 & 28.34 & 32.94 & 0.9987 & 0.9985 \\
\hline 1.2 & 2.3 & 88.9 & 30.8 & 47.52 & 44.59 & 28.64 & 33.25 & 0.9993 & 0.9991 \\
\hline 0.2 & 1.7 & 72.8 & 24.9 & 55.63 & 45.92 & 29.51 & 34.17 & 0.9995 & 0.9994 \\
\hline 0.0 & 0.0 & 121.2 & 40.4 & Inf & Inf & 27.29 & 32.07 & 0.9999 & 0.9999 \\
\hline 0.0 & 0.0 & 158.2 & 52.7 & Inf & Inf & 26.14 & 30.91 & 0.9999 & 0.9999 \\
\hline 0.0 & 0.0 & 21.4 & 7.1 & Inf & Inf & 34.83 & 39.60 & 1.0000 & 1.0000 \\
\hline 0.0 & 0.0 & 0.0 & 0.0 & Inf & Inf & Inf & Inf & 1.0000 & 1.0000 \\
\hline
\end{tabular}


Table 3. Performance Comparison on \#10 McM Image

\begin{tabular}{|r|r|r|r|c|c|c|c|c|c|}
\hline \multicolumn{1}{|c|}{$\mathrm{M}_{1}$} & \multicolumn{1}{c|}{$\mathrm{M}_{2}$} & \multicolumn{1}{c|}{$\mathrm{M}_{3}$} & $\mathrm{M}_{4}$ & $\mathrm{M}_{5}$ & $\mathrm{M}_{6}$ & $\mathrm{M}_{7}$ & $\mathrm{M}_{8}$ & $\mathrm{M}_{9}$ & $\mathrm{M}_{10}$ \\
\hline 156.3 & 149.4 & 146.8 & 150.9 & 26.19 & 26.39 & 26.46 & 26.35 & 0.9715 & 0.9692 \\
\hline 156.5 & 149.9 & 148.8 & 151.7 & 26.19 & 26.37 & 26.40 & 26.32 & 0.9764 & 0.9747 \\
\hline 152.3 & 159.1 & 160.9 & 157.4 & 26.30 & 26.11 & 26.07 & 26.16 & 0.9874 & 0.9864 \\
\hline 189.5 & 197.7 & 194.7 & 194.0 & 25.35 & 25.17 & 25.24 & 25.25 & 0.9870 & 0.9863 \\
\hline 180.8 & 99.4 & 77.9 & 119.4 & 25.56 & 28.16 & 29.21 & 27.36 & 0.9936 & 0.9930 \\
\hline 115.8 & 184.3 & 113.4 & 137.8 & 27.49 & 25.48 & 27.59 & 26.74 & 0.9877 & 0.9874 \\
\hline 96.2 & 78.7 & 234.2 & 136.4 & 28.30 & 29.17 & 24.43 & 26.78 & 0.9829 & 0.9823 \\
\hline 12.4 & 71.7 & 126.6 & 70.3 & 37.19 & 29.57 & 27.11 & 29.66 & 0.9928 & 0.9926 \\
\hline
\end{tabular}

Table 4. Performance Comparison on \#11 McM Image

\begin{tabular}{|c|c|c|c|c|c|c|c|c|c|}
\hline $\mathrm{M}_{1}$ & $\mathrm{M}_{2}$ & $\mathrm{M}_{3}$ & $\mathrm{M}_{4}$ & $\mathrm{M}_{5}$ & $\mathrm{M}_{6}$ & $\mathrm{M}_{7}$ & $\mathrm{M}_{8}$ & $\mathrm{M}_{9}$ & $\mathrm{M}_{10}$ \\
\hline 153.9 & 154.9 & 154.3 & 154.4 & 26.26 & 26.23 & 26.25 & 26.24 & 0.9692 & 0.9670 \\
\hline 156.2 & 146.0 & 156.4 & 152.9 & 26.19 & 26.49 & 26.19 & 26.29 & 0.9746 & 0.9728 \\
\hline 141.2 & 153.6 & 131.0 & 141.9 & 26.63 & 26.27 & 26.96 & 26.61 & 0.9834 & 0.9821 \\
\hline 158.5 & 159.5 & 245.3 & 187.8 & 26.13 & 26.10 & 24.23 & 25.39 & 0.9904 & 0.9896 \\
\hline 175.4 & 151.2 & 265.3 & 197.3 & 25.69 & 26.33 & 23.89 & 25.18 & 0.9925 & 0.9918 \\
\hline 183.6 & 157.8 & 272.6 & 204.7 & 25.49 & 26.15 & 23.78 & 25.02 & 0.9924 & 0.9919 \\
\hline 224.6 & 183.7 & 260.4 & 222.9 & 24.62 & 25.49 & 23.97 & 24.65 & 0.9881 & 0.9877 \\
\hline 253.8 & 242.2 & 301.1 & 265.7 & 24.09 & 24.29 & 23.34 & 23.89 & 0.9749 & 0.9748 \\
\hline
\end{tabular}

Note that inf represents infinity.

\section{Conclusions}

In this paper, we studied on bit plane watermarking. Watermarking applications have been studied to protect digital image against illegal copyright violation. Generally speaking, adding watermarking symbol in least significant bit (LSB) is desirable. We showed how to design watermarking application, and compared watermarking assessment on different bit plane. Two datasets were used: Kodak and McM datasets.

\section{Acknowledgements}

This research was supported by Basic Science Research Program through the National Research Foundation of Korea (NRF) funded by the Ministry of Science, ICT and Future Planning (2014025627).

\section{References}

[1] P. Kumsawat, K. Attakitmongcol and A. Srikaew, "A New Approach for Optimization in Image Watermarking by Using Genetic Algorithms", IEEE Trans. on Signal Processing, vol. 53, no. 12, (2005) December, pp. 4707-4719.

[2] S. Chen and H. Leung, "Ergodic Chaotic Parameter Modulation With Application to Digital Image Watermarking", IEEE Trans. on Image Processing, vol. 14, no. 10, (2005) October, pp.1590-1602.

[3] I. J. Cox, J. Kilian, F. T. Leighton and T. Shamoon, "Secure Spread Spectrum Watermarking for Multimedia", IEEE Trans. on Image Processing, vol. 6, no. 12, (1997) December, pp. 1673-1686.

[4] L. Ghouti, A. Bouridane, M. K. Ibrahim and S. Boussakta, "Digital Image Watermarking Using Balanced Multiwavelets", IEEE Trans. on Signal Processing, vol. 54, no. 4, (2006) April, pp.1519-1536, April, 2006.

[5] Y. Fang, "Analysis on crossover probability estimation using LDPC syndrome," Science China: Inf. Sci., vol. 54, no. 9, (2011) September, pp. 1895-1904. 
[6] Y. Fang, "Joint source-channel estimation using accumulated LDPC syndrome," IEEE Commun. Lett., vol. 14, no. 11, (2010) November, pp. 1044-1046.

[7] Y. Fang, "EREC-based length coding of variable-length data blocks," IEEE Trans. Circ. Syst. Video Technol., vol. 20, no. 10, (2010) October, pp. 1358-1366.

[8] Y. Fang, "Distribution of distributed arithmetic codewords for equiprobable binary sources," IEEE Signal Process. Lett., vol. 16, no. 12, (2009) December, pp. 1079-1082.

[9] Y. Fang, "Crossover probability estimation using mean-intrinsic-LLR of LDPC syndrome," IEEE Commun. Lett., vol. 13, no. 9, (2009) September, pp. 679-681.

[10] Y. Fang, G. Jeon and Jechang Jeong, "State-information-assisting EREC," IEEE Signal Process. Lett., vol. 16, no. 4, (2009) April, pp. 260-263.

[11] Y. Fang and Jechang Jeong, "Correlation parameter estimation for LDPC-based Slepian-Wolf coding," IEEE Commun. Lett., vol. 13, no. 1, (2009) January, pp. 37-39.

[12] Y. Fang, J. Jeong, et al., "Error detection based on MB types," Science China: Inf. Sci., vol. 51, no. 4, (2008) April, pp. 433-439.

[13] Y. Fang, J. Jeong, et al., "Robust video transmission using block-interleaved error resilient entropy coding," SPIE Optical Engineer., vol. 46, no. 12, (2007) December, 127401-1-7.

[14] Y. Fang, C. Wu, et al., "Video transmission using advanced partial backward decodable bit stream," Elsevier J. Visual Commun. \& Image Represent., vol. 18, no. 2, (2007) April, pp. 186-190.

[15] Y. Fang, C. Wu, et al., "Bi-directional error resilient entropy coding (BEREC)," EURASIP Signal Process.: Image Commun., vol. 21, no. 8, (2006) September, pp. 647-652.

[16] G. Jeon, M. Anisetti, V. Bellandi and J. Jeong, "Fuzzy Rule-Based Edge-Restoration Algorithm in HDTV Interlaced Sequences", IEEE Transactions on Consumer Electronics, (2007) May.

[17] G. Jeon, M. Anisetti, V. Bellandi, E. Damiani and J. Jeong, "Rough Sets- Assisted Subfield Optimization for Alternating Current Plasma Display Panel", IEEE Transactions on Consumer Electronics, (2007) August.

[18] G. Jeon, M. Anisetti, V. Bellandi, E. Damiani and J. Jeong "Fuzzy Weighted Approach to Improve Visual Quality of Edge-Based Filtering" IEEE Transactions on Consumer Electronics, (2007) November.

[19] G. Jeon, M. Anisetti, D. Kim, V. Bellandi, E. Damiani and J. Jeong, "Fuzzy rough sets hybrid scheme for motion and scene complexity adaptive deinterlacing", IMAVIS Image and Vision Computing Journal, Elsevier ISSN: 0262-885, vol. 27, no. 4, (2009) March, pp. 425-436.

[20] G. Jeon, M. Anisetti, V. Bellandi, E. Damiani and J. Jeong, "Designing of a type-2 fuzzy logic filter for improving edge-preserving restoration of interlaced-to-progressive conversion", Information Sciences, Elsevier, Giugno, (2009).

[21] G. Jeon, M. Anisetti, J. Lee, V. Bellandi, E. Damiani and J. Jeong, "Concept of Linguistic Variable-Based Fuzzy Ensemble Approach: Application to Interlaced HDTV Sequences", IEEE Transactions on Fuzzy Systems, IEEE, (2009) December.

[22] G. Jeon, S. J. Park, Y. Fang, M. Anisetti, V. Bellandi, E. Damiani and J. Jeong, "Specification of efficient block matching scheme for motion estimation in video compression," SPIE Optical Engineering, vol. 48, no. 12, (2009) December.

[23] G. Jeon, M. Y. Jung, M. Anisetti, V. Bellandi, E. Damiani and J. Jeong, "Specification of the Geometric Regularity Model for Fuzzy If-Then Rule-Based Deinterlacing", IEEE JOURNAL OF DISPLAY TECHNOLOGY, Vol. 6, no. 4, (2010) April.

[24] G. Jeon, M. Anisetti and S. Kang, "A Rank-Ordered Marginal Filter for Deinterlacing” Sensors, (2013).

[25] N. G. Rao, T. Sravani and V. V. Kumar, "OCRM: Optimal Cost Region Matching Similarity Measure for Region Based Image Retrieval,” IJMUE, vol. 9, no. 4, (2014) April, pp. 327-342.

[26] L. Shen and Y. Niu, "Blind Color Image Fusion Based on the Optimal Multi-objective Particle Swarm Optimization," IJMUE, vol. 2, no. 3, (2007) July, pp. 51-62.

[27] B.-H. Kang, “A Review on Image and Video processing,” IJMUE, vol. 2, no. 2, (2007) April, pp. $49-64$.

[28] S.-S. Yoo, Y.-T. Kim, S.-J. Youk, J.-H. Kim and B.-K. Lee, "Adaptive-Binning Color Histogram for Image Information Retrieval,” IJMUE, vol. 1, no. 4, (2006) December, pp. 45-53.

\section{Author}

Gwanggil Jeon, is currently an assistant professor with the Department of Embedded Systems Engineering, Incheon National University, Incheon, Korea. His research interests fall under the umbrella of image processing, particularly image compression, motion estimation, demosaicking, and image enhancement as well as computational intelligence such as fuzzy and rough sets theories. 